\title{
COMPLICATIONS DURING DIAGNOSTIC CARDIAC CATHETERISATION IN CHILDREN WITH TETRALOGY OF FALLOT
}

\author{
Y. Singh ${ }^{1,2}$, J. Thomson ${ }^{2}$
}

${ }^{1}$ Department of Neonatal Medicine, Bradford Teaching Hospitals NHS Trust, Bradford, ${ }^{2}$ Department of Paediatric Cardiology, Leeds Teaching Hospitals NHS Trust, Leeds, UK

Background: Diagnostic cardiac catheterisation is an important tool to define the detailed anatomy in children with Fallot's tetralogy pre-operatively. With advance in technology most of this information can be gained by cardiac echocardiogram or further non-invasive imaging like CT scan or MRI. A recent telephone survey across the cardiac units in the UK showed that majority centers now use cardiac catheterisations as a secondary investigation. Diagnostic cardiac catheterisation still remains extremely important in selected cases.

Aims and objectives: To find out the risk of significant complications during or within 12 hours of routine diagnostic catheterisation in cases of Fallot's Tetralogy.

Design and methods: A retrospective observational study and cases were identified from the database. Data was collected from 72 Fallot's cases undergoing diagnostic cardiac catheterisation at Leeds General Infirmary between January 2004 and September 2009.

Results: 17 out of the $72(23 \%)$ cases had significant complication as a result of diagnostic cardiac catheterisation. Common complications included hypercyanotic spells in 10 cases (14\%) and heart block in 4 (5\%) cases. Other complications included ventricular ectopics (1 case), cold leg requiring heparin ( 1 case) and significant bradycardia ( 1 case) requiring external cardiac message.

Conclusion: Diagnostic cardiac catheterisation in small infants and children with Tetralogy of Fallot's carries a high risk of complications even in expert hands. In our series $23 \%$ had significant complications. Whenever possible detailed anatomy should be defined by other non-invasive modalities like echocardiogram, MRI scan etc and cases for diagnostic cardiac catheterisation should be chosen judiciously. 